Original article

\title{
Hepatic cirrhosis according to the forensic medical autopsy data from Moscow for the period of 2007-2016
}

\author{
Yuri I. Pigolkin, Yuri E. Morozov, Irina V. Globa, Vladimir N. Nikolenko \\ I.M. Sechenov First Moscow State Medical University (Sechenov University), Moscow, Russia
}

Received 14 November 2018, Revised 8 August 2019, Accepted 02 November 2019

(C) 2018, Pigolkin Yu.I., Morozov Yu.E., Globa I.V., Nikolenko V.N.

(C) 2018, Russian Open Medical Journal

\begin{abstract}
Study purpose is to demonstrate the dynamics of mortality due to hepatic cirrhosis according to forensic medical autopsy data over the period from 2007 to 2016.

Material and methods - 268,139 protocols of forensic autopsies were studied to identify the number of deaths by hepatic cirrhosis over ten years between 2012 and 2016 and to determine the number of cases of liver failure associated with cirrhosis. To clarify the dynamics of mortality due to hepatic cirrhosis, a comparative analysis of two chronologically consecutive five-year periods, 2007-2011 and 2012-2016, has been conducted. The age of deceased by hepatic cirrhosis was studied. The number of cases with a histologically verified form of hepatic cirrhosis was specified. The average values and the percentage ratio of the number of examinations performed to the total number of all autopsies for non-violent death cases were calculated.

Results - Over ten years of study, death by hepatic cirrhosis was diagnosed 3,092 times, including 749 times with histologically confirmed liver failure. In 2009, there was an abrupt increase in mortality due to hepatic cirrhosis by the factor of 3.8, and the lowest mortality level was found in 2016. When comparing the mentioned two quinquennia, the first of them, 2007-2011, was characterized by an increase in the number of diagnosed hepatic cirrhosis. During the second one, 2012-2016, on the contrary, the dynamics of a decrease in the number of hepatic cirrhosis was revealed. At the average, the annual decrease in the number of hepatic cirrhosis diagnosed during the forensic medical examinations of corpses was $7.7 \%$. The number of established cases of liver failure during the first five-year period compared with the data on the second one was significantly greater. In 2010, there were 109 such cases, in 2016 only 13. Among the histologically verified diagnoses, micronodular and mixed forms of hepatic cirrhosis prevailed. Most of the cases of hepatic cirrhosis were found in men aged within 31-40.

Conclusion - It has been found that over the past five years there has been a shift in the forensic-medical mortality values due to hepatic cirrhosis from the increasing trend to the decreasing one. If within the period of 2007-2011 an increase in diagnosed hepatic cirrhosis was observed, then during 2012-2016 a pronounced decreasing tendency in the number of such diagnoses was disclosed. During that time the number of histologically verified cases of hepatic failure decreased by $43.0 \%$. As a cause of death, hepatic cirrhosis was more often recorded in men aged within 30-40.
\end{abstract}

Keywords: hepatic cirrhosis, liver failure, mortality values, protocols of forensic medical autopsies.

Cite as Pigolkin Yul, Morozov YuE, Globa IV, Nikolenko VN. Hepatic cirrhosis according to the forensic medical autopsy data from Moscow for the period of 2007-2016. Russian Open Medical Journal 2019; 8: e0412.

Correspondence to Yuri I. Pigolkin. Address: Sechenov First Moscow State Medical University (Sechenov University), Rossolimo str. 15/13, build. 2, Moscow, 119021, Russia. Phone: +79161462201. E-mail: pigolkin@mail.ru.

\section{Introduction}

The structure of data on causes of non-violent death, constituting approximately $60 \%$ of the total number of forensic medical autopsies, is the subject of a study of forensic medical science and practice. Death by hepatic cirrhosis is a type of nonviolent death [1]. In economically developed countries, hepatic cirrhosis is among the six top causes of death at the age of 35-60, occurring with a frequency of 14 to 30 cases per 100,000. According to the literature five years ago, there are increasing rates of morbidity and mortality due to hepatic cirrhosis [2, 3]. In Russia, the incidence of hepatic cirrhosis is in the first place. In 2010, it increased by $73.8 \%$, and in St. Petersburg it reached 124.4 incidents per 100 thousand, which is 3 times higher than the average in
Russia. This problem is exacerbated by the high prevalence of drug addiction and indiscriminate unprotected sex $[4,5]$.

Forensic medical diagnosis of hepatic cirrhosis and concomitant liver failure is based mainly on the results of autopsy studies and histological examinations, which deal with characteristic pathological changes in the liver. In the annual statistical forensic medical reports, data on mortality due to hepatic cirrhosis are summarized in the section of diseases of the digestive system. Therefore, hepatic cirrhoses, even complicated by end-stage liver failure, remain beyond the focus the statistical registration. No publications are comparing clinical and forensic medical indices of morbidity and mortality due to hepatic cirrhosis and the number of cases of severe liver failure established at that. In most foreign countries, cases of sudden death, including from 
hepatic cirrhosis, are subject to pathological examination instead of a forensic medical one.

All these determine the topicality of the investigation of domestic forensic medical reports, the protocols of which contain exclusive information about hepatic cirrhosis and the severity of liver failure caused by it. The obtained results can be used as a comparison object with the indices of the corresponding clinical data for analysis and conclusions about the completeness and validity of the forensic medical diagnosis of hepatic cirrhosis and liver failure.

\section{Material and Methods}

The materials of the study were the results of 268,139 forensic medical autopsies over 10 years (2007-2016) in Moscow, in which the death by hepatic cirrhosis amounted to 3092 cases, and by liver failure caused by hepatic cirrhosis made up 749 cases. The objects of the study were annual reports on the activities of the Bureau of Forensic Medical Examination (Moscow, Russia), acts of forensic medical expert examinations of corpses and acts of forensic histological examinations, the content of autopsy and histological diagnoses as well as their written justifications. Data from experts' opinions, autopsy protocols and acts of inspections have been summarised.

All cases of hepatic cirrhosis diagnosed during forensic medical examination of corpses were grouped by the calendar year and the two consecutive five-year periods, depending on the established morphological form of the disease. According to the pathomorphological classification based on the diameter of the connective tissue nodes, there are three main forms of hepatic cirrhosis [6] as follows: 1) micronodular form of hepatic cirrhosis (MNHC), characterised by a diameter of connective tissue nodes of less than 3 millimeters (most nodes are usually in range of 1-3 $\mathrm{mm}$ ); 2) coarse nodular form of hepatic cirrhosis (CNHC) with a diameter of nodes of more than $3 \mathrm{~mm}$; 3 ) mixed hepatic cirrhosis $(\mathrm{MHC})$, in which there are both small and large connective tissue nodes in the liver parenchyma.

Additionally, a data sampling in acts of forensic histological examinations together with a grouping of objective findings characterizing the stage of liver failure according to the severity of destructive and dystrophic changes in structure- functional sections of hepatic acini have been carried out. According to morphological data, the stage of liver failure indicates the prevalence of destructive and dystrophic changes of hepatocytes in the hepatic acini. Pursuant to this concept, all deaths by hepatic cirrhosis were divided into three groups, each of which corresponded to a certain stage of liver failure [7] as follows:

i) Compensated stage of liver failure (CSLF) with initial morphological changes, when during histological examination destructive and dystrophic changes of hepatocytes are detected only in the hepatoportal sections of the hepatic acini;

ii) Decompensated stage of liver failure (DSLF) or a stage of pronounced changes, when destructive and dystrophic changes are detected throughout both the hepatoportal sections and hepatic tubules of the hepatic acini;

iii) The end-stage of liver failure (ESLF) when during histological examination destructive and dystrophic changes are revealed totally in the hepatocytes of all three sections of the hepatic acini: hepatoportal, tubular and the central vein area.

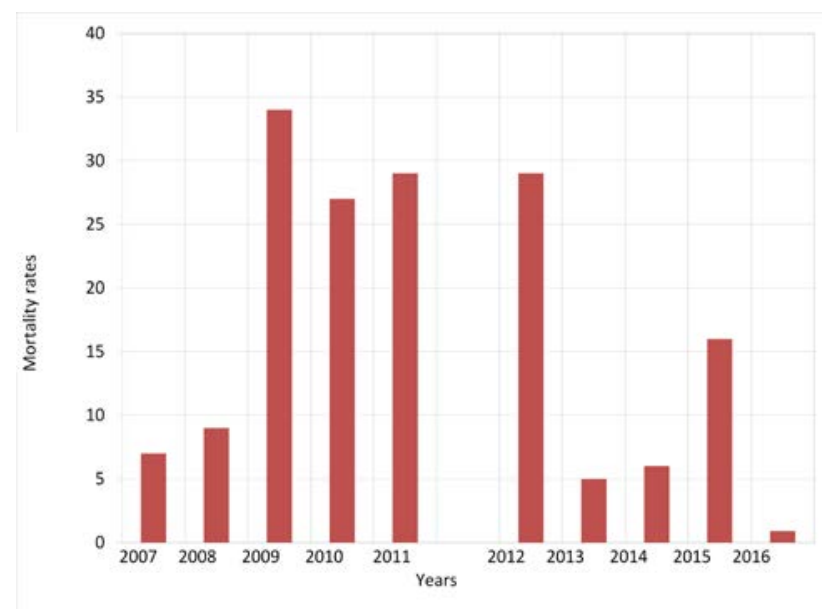

Figure 1. Mortality rates from the liver cirrhosis (\%) according to the number of cases of non-violent dearth in 2007-2016.

The data on the number of cases of hepatic cirrhosis is given, taking into account the pathological form and stage of liver failure in the indicated chronological periods. Mortality values from hepatic cirrhosis are expressed on a percentage basis to the total number of all autopsies for non-violent death for each calendar year of the ten-year period studied. Annual and five-year figures were compared and the trend and dynamics of shifts in mortality from hepatic cirrhosis were determined. The age composition of the deceased in age groups 20-30, 30-40, 41-45, 46-50, 51-55, 5660 and 61-70 was scrutinised.

\section{Results}

When analyzing the obtained results, it turned out that the number of diagnoses of hepatic cirrhosis established according to the data of forensic autopsies in Moscow during the first quinquennium, 2007-2011, increased by a factor of 3.9. The largest number of deaths was recorded within 2009-2012 (Figure 1).

During the second quinquennium, beginning in 2013, the number of diagnosed cases of hepatic cirrhosis, on the contrary, decreased. The peak of mortality occurred in 2012, and the lowest number of deaths by hepatic cirrhosis occurred in 2016.

Among hepatic cirrhoses, the micronodular and mixed pathological forms of the disease predominated (Table 1 ).

Table 1. The morphological forms of hepatic cirrhosis according to forensic medical autopsies in Moscow within the period of 2007-2016

\begin{tabular}{lccc}
\hline & The number of cases of the morphological forms of hepatic \\
cirrhosis & & MHC \\
Years & MNHC & CNHC & 91 \\
\hline 2007 & 35 & 7 & 98 \\
2008 & 58 & 15 & 507 \\
2009 & 109 & 29 & 313 \\
2010 & 166 & 34 & 353 \\
2011 & 143 & 55 & 200 \\
2012 & 255 & 94 & 76 \\
2013 & 7 & 12 & 70 \\
2014 & 18 & 26 & 139 \\
2015 & 80 & 85 & 9 \\
2016 & 4 & 4 & \\
\hline
\end{tabular}

MNHC, micronodular hepatic cirrhosis; CNHC, coarse nodular hepatic cirrhosis; MHC, mixed hepatic cirrhosis. 
Table 2. The detection frequency of liver failure according to the forensic medical autopsies in Moscow from 2007 to 2016

\begin{tabular}{lccc}
\hline & \multicolumn{1}{c}{ The number of cases of liver failure by stages } \\
Years & CSLF & DSLF & ESLF \\
\hline 2007 & 1 & 27 & 34 \\
2008 & 0 & 53 & 41 \\
2009 & 2 & 55 & 48 \\
2010 & 5 & 54 & 50 \\
2011 & 2 & 48 & 57 \\
2012 & 3 & 57 & 69 \\
2013 & 1 & 10 & 27 \\
2014 & 3 & 12 & 19 \\
2015 & 2 & 25 & 31 \\
2016 & 0 & 6 & 7 \\
\hline
\end{tabular}

CSLF, compensated stage of liver failure; DSLF, decompensated stage of liver failure; ESLF, end-stage of liver failure.

During each year of the decade-long period studied, the number of mixed and micronodular forms of liver failure significantly prevailed over the number of coarse nodular forms of the disease. However, during the second quinquennium compared with the first one, the frequency of occurrence of coarse nodular forms increased almost 2 times.

Over 10 years liver failure was diagnosed 749 times, the highest number of cases of this illness, 109, was established in 2010. In 2016, the number of histologically verified forensic medical diagnoses of liver failure decreased to 13 cases (Table 2).

In the vast majority of observations, in the condition of hepatic cirrhosis, the end-stages and decompensated stages of liver failure prevailed. When intercomparing the two quinquennia by the number of histologically verified cases of liver failure, it turned out that in the second quinquennium, 2012-2016, the number of such cases was down by $43.0 \%$.

It is turned out that the largest number of observations, $47 \%$ of deceased by hepatic cirrhosis, was accounted for by the age group of 31-40 years.

\section{Discussion}

The noted distinction of hepatic cirrhosis is a high mortality. According to the available publications, a clear increase in the number of deaths by hepatic cirrhosis in our country was observed from 2007 to 2012, which was associated by the authors with excessive consumption of alcohol and drugs at that time. The peak of mortality accounted for by 2009-2010, and in 2013 a decrease in the number of deaths by this disease [8-10] was observed. Our studies confirm these literature data since from 2007 through 2011 and in 2012 we established high figures of mortality due to hepatic cirrhosis.

According to our results, in Moscow over the 10-year period of forensic autopsies, wave-like shifts in mortality due to hepatic cirrhosis were observed. The maximum peak of mortality occurred in 2009, which was characterized by the growth of drug addiction. Since 2013, the number of deaths by hepatic cirrhosis decreased in parallel with the decrease in the total number of forensic autopsies. Thus, during the second quinquennium, 2013 to 2016, we identified a new trend in the dynamics of mortality due to hepatic cirrhosis, which consists in reducing the number of deaths by hepatic cirrhosis. The change in the mortality trend due to hepatic cirrhosis is a new piece of information that is not reflected in recent publications.
At death by hepatic cirrhosis, the decompensated stage and end-stage of liver failure prevailed. The most important factor in the genesis of alcoholic hepatic cirrhosis is the necrosis of hepatocytes due to the direct toxic effects of alcohol, as well as autoimmune processes. The condition of hepatic cirrhosis has been forming for months or even years. Over this time, the gene apparatus of hepatocytes changes and generations of pathologically altered cells are developed. This process in the liver, characterized as immune inflammatory one, is logically explained from the standpoint of structural and functional shifts in the liver acinus under hepatic cirrhosis. Destructive and dystrophic shifts detected by histological examination of the liver in the hepatoportal and tubular sections of the liver acini, as well as in the central sections of the liver cells, are characterized by successively aggravated stages of liver failure [7, 11-13]. The acinar model facilitates the understanding of the various clinical and morphological characteristics of liver failure. It is noteworthy that pathological changes identified by us in the end-stage of liver failure and individual clinical and laboratory stigmas characterizing severe exogenous intoxications show signs that from different sides equally characterize the same state, hepatic coma [14].

In the second quinquennium, 2012-2016, when performing forensic autopsies, a significant decrease (by $43.0 \%$ ) in the number of cases with a histologically verified stage of liver failure was revealed. This phenomenon requires additional comprehension and understanding to clarify the reasons for such significant shifts. It is not unlikely that the decrease in the number of established cases of liver failure is determined by a decrease in the frequency of alcoholic excesses associated with sudden death [1] or a consequence of the modified spectrum of reasons for prescribing a forensic medical examination of a corpse in cases of death at hospital [3].

The main scientific interest of the study conducted is the ability to assess the dynamics of mortality from hepatic cirrhosis and concomitant liver failure according to data from primary forensic medical acts of corpse autopsies and acts of forensic histological examinations.

\section{Conclusion}

A comparative analysis of the forensic medical figures of mortality within the periods of 2007-2011 and 2012-2016 allowed revealing a new trend of decreasing mortality due to hepatic cirrhosis over the past five years in Moscow (Russia).

\section{Limitations}

In this study, far not all factors determining the morbidity and mortality due to hepatic cirrhosis and liver failure were investigated. These were population differences, concomitant chronic diseases, intoxications and many others. The study of the influence of these factors should be the subject of further studies in this field.

It was not the purpose of this communication to evaluate the correctness of the forensic medical reports. A special procedural order regulating the rules for the performing additional and repeated examinations is devoted to this issue.

Conflict of interest: none declared. 


\section{References}

1. Kovalev AV, Morozov YuE, Samokhodskaya OV, Bereznikov AV Alcoholassociated mortality in Russia (based on the materials for the period from 2011 till 2016). Sud Med Ekspert 2017; 60(6): 4-8. Russian. https://doi.org/10.17116/sudmed20176064-8.

2. Wang $\mathrm{Y}, \mathrm{Hou} \mathrm{JL}$. Fibrosis assessment: impact on the current management of chronic liver disease and application of quantitative invasive tools. Hepatol Int 2016; 10(3): 448-461. https://doi.org/10.1007/s12072-015-9695-0.

3. Pavlov ChS, Kuznetsova EA, Arslanyan MG, Semenistaya MCh, Glushenkov DV, Nikolenko VN. Non-alcoholic fatty li ver disease: modern concepts of etiology, pathogenesis, diagnostics and treatment. Medical news of North Caucasus 2017; 12(2): 230-234. Russian. https://doi.org/10.14300/mnnc.2017.12066.

4. Morozov YuE, Mazus Al, Shigeev SV. The HIV-associated diseases encountered in the practice of forensic medical autopsies. Sud Med Ekspert 2018; 61(4): 28-31. Russian. https://doi.org/10.17116/sudmed201861428.

5. Kil'dyushov EM, Kovalev AV, Morozov YuE, Mazus Al, Serebryakov EM, Kudimov IN, et al. Monitoring HIV infection morbidity in Moscow based on the data of forensic medical expertise. Sud Med Ekspert 2015; 58(5): 4-8. Russian. https://doi.org/10.17116/sudmed20155854-8.

6. Liu H, Shao Y, Guo D, Zheng Y, Zhao Z, Qiu T Cirrhosis Classification Based on Texture Classification of Random Features. Comput Math Methods Med 2014; 2014: 536308. https://doi.org/10.1155/2014/536308.

7. Morozov YuE, Porodenko VA, Travenko EN, Gornostaev DV. Morphological markers of liver function in alcohol intoxication. Sud Med Ekspert 2019; 62(3): 34-41. Russian. https://doi.org/10.17116/sudmed20196203137.

8. Durand F, Valla D. Assessment of the prognosis of cirrhosis. Semin Liver Dis 2008; 28(1): 110-122. https://doi.org/10.1055/s-2008-1040325.

9. Glantz SA. Primer of biostatistics. Fourth edition. NY, USA: McGrawHill, Health Professions Division, 1997; 473 p. https://books.google.ru/books/about/Primer_of Biostatistics.html?id =XrdpAAAAMAAJ\&redir esc $=\mathrm{y}$

10. Ivashkin VT, Mayevskaya MV. Alcohol-viral liver disease. Moscow, Russia: Litterra, 2007; 160 p. Russian.

11. Ergelen R, Yilmaz Y, Asedov R, Celikel C, Akin H, Bugdayci O, et al. Comparison of Doppler ultrasound and transiente inpatients with nonalcoholic steatohepatitis. Abdom Radiol 2016; 41(8): 1505-1510. https://doi.org/10.1007/s00261-016-0699-6.

12. Shahgildyan IV, Yasinskii A.A, Mihailov MI, Ershova ON, Lytkina IN, SHulakova NI, et al. Characteristics of chronic parenteral viral hepatitis in the Russian Federation. Justification of need and assessment hepatitis vaccination efficacy $A$ and $B$ patients with chronic diseases liver. Journal Infectology 2010; 2(4): 127-128. Russian. https://journal.niidi.ru/jofin/article/view/321/318.

13. Clinical dilemmas in the non-alcoholic fatty liver disease. 1st edition. R. Williams, S.D. Taylor-Robinson, Eds. Wiley-Blackwell, 2016; 256 p. https://doi.org/10.1002/9781118924938.

14. Morozov YE, Velenko PS. The characteristic of protein biosynthesis in brain neurons with chronic alcohol intoxication. Sud Med Ekspert 2018; 61(1): 49-51. https://doi.org/10.17116/sudmed201861149-51.

\section{Authors:}

Yuri I. Pigolkin - Corresponding Member of the Russian Academy of Sciences, MD, DSc, Professor, Head of Forensic Medicine Department, Sechenov First Moscow State Medical University (Sechenov University), Moscow, Russia. https://orcid.org/0000-0001-5370-4931.

Yuri E. Morozov - MD, DSc, Associate Professor, Forensic Medicine Department, Sechenov First Moscow State Medical University (Sechenov University), Moscow, Russia. https://orcid.org/0000-0002-0594-257X.

Irina V. Globa - MD, Postgraduate, Department of Forensic Medicine Department, Sechenov First Moscow State Medical University (Sechenov University), Moscow, Russia. https://orcid.org/0000-0002-7185-4324.
Vladimir N. Nikolenko - MD, DSc, Professor, Head of Human Anatomy department, Sechenov First Moscow State Medical University (Sechenov University), Moscow, Russia. https://orcid.org/0000-0001-9532-9957. 